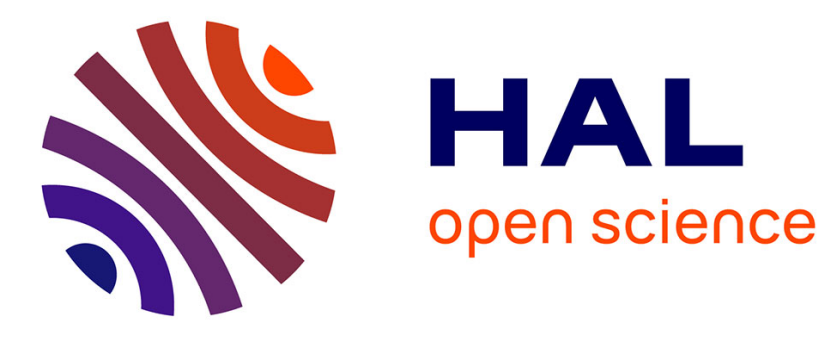

\title{
Towards practical large-eddy simulations of complex turbulent flows
}

Jérôme Boudet, Adrien Cahuzac, Pierre Borgnat, Emmanuel Lévêque, Federico Toschi

\section{- To cite this version:}

Jérôme Boudet, Adrien Cahuzac, Pierre Borgnat, Emmanuel Lévêque, Federico Toschi. Towards practical large-eddy simulations of complex turbulent flows. 12th EUROMECH European Turbulence Conference, Sep 2009, Marburg, Germany. ensl-00420589

\section{HAL Id: ensl-00420589}

\section{https://hal-ens-lyon.archives-ouvertes.fr/ensl-00420589}

Submitted on 29 Sep 2009

HAL is a multi-disciplinary open access archive for the deposit and dissemination of scientific research documents, whether they are published or not. The documents may come from teaching and research institutions in France or abroad, or from public or private research centers.
L'archive ouverte pluridisciplinaire HAL, est destinée au dépôt et à la diffusion de documents scientifiques de niveau recherche, publiés ou non, émanant des établissements d'enseignement et de recherche français ou étrangers, des laboratoires publics ou privés. 


\title{
Towards practical large-eddy simulations of complex turbulent flows
}

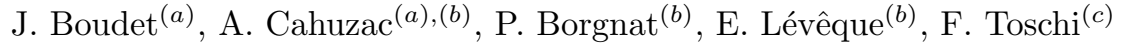 \\ (a) Laboratoire de Mécanique des Fluides et d'Acoustique, Ecole Centrale de \\ Lyon, Université de Lyon, CNRS, France \\ (b) Laboratoire de Physique, École normale supérieure de Lyon, Université de \\ Lyon, CNRS, France \\ (c) Technische Universiteit Eindhoven, The Netherlands \\ emmanuel.leveque at ens-lyon.fr
}

Turbulence that occurs in nature, or in engineering flows, is usually not, even approximatively, homogeneous. There are frequent variations of the mean velocity with position. By explicating the scale-by-scale energy budget of nonhomogeneous turbulence, it has been argued that the subgrid-scale (SGS) stress tensor should encompass two types of interactions [1,2]: (i) between the mean velocity and the resolved fluctuating velocities (the rapid part of the SGS stress) and (ii) among the resolved fluctuating velocities themselves (the slow part of the SGS stress). The rapid part is related to the large-scale distortion, while the slow part is associated with the Kolmogorov's energy cascade [3]. Interestingly, these developments end up with a shear-improved Smagorinsky model (SISM) [1], for which the SGS viscosity writes

$$
\nu_{\mathrm{sgs}}=\left(C_{s} \Delta\right)^{2}(|\overline{\mathrm{S}}|-\mathcal{S}) .
$$

$C_{s} \simeq 0.2$ is the Smagorinsky constant, $\Delta$ is the grid size and $|\overline{\mathrm{S}}| \equiv\left(2 \overline{\mathrm{S}}_{i j} \overline{\mathrm{S}}_{i j}\right)^{1 / 2}$ is the norm of the resolved rate-of-strain. Finally, $\mathcal{S} \equiv|\langle\mathrm{S}\rangle|$ is the characteristic shear associated with the mean flow. In practical simulations, $\mathcal{S} \simeq|\langle\overline{\mathrm{S}}\rangle|$.

Interestingly, the SISM does not call for any adjustable parameter nor adhoc damping function. It does not use any kind of dynamic adjustment either. However, a special care must be taken in estimating the ensemble-averaged rate-of-strain, $\langle\overline{\mathrm{S}}\rangle$. Ensemble average may be replaced by space average over homogeneous directions (whenever it is possible) or time average. This is adapted to simple-geometry flows or (statistically) stationary turbulent flows. This was the case of our first tests (which focused on SGS modeling errors) concerning a bi-periodic plane-channel flow [1] and a backward-facing step flow [4]. These results are promising as they indicate that the SISM possesses a predictive capacity essentially equivalent to the dynamic Smagorinsky model [5] but with a computational cost and a manageability comparable to the Smagorinsky model [6]. 
It is now our motivation to examine how the SISM behaves in CFD solvers, which usually rely on a coarser grid and a lower-order discretization scheme, and apply to complex-geometry unsteady flows [8]. A procedure is introduced to evaluate the mean flow from the running velocity field. Our proposal is based on temporal smoothing that highlights longer-term trends or cycles but erases short-term fluctuations. Our physical assumption is that the mean velocity is given by the "low-frequency component" of the velocity, and that the turbulent part of the velocity adds itself to this "unsteady mean". An exponentially-weighted moving average (or exponential smoothing) is considered.

At a given grid-point, the idea behind exponential smoothing is to update (at each time step) the previous estimate of the mean by taking into account the new instantaneous value. Let $u^{(n)}$ stand for a component of velocity and $[u]^{(n)}$ the estimated mean, at time $n$. The update writes

$$
[u]^{(n+1)}=\left(1-c_{\text {exp. }}\right)[u]^{(n)}+c_{\text {exp. }} u^{(n+1)}
$$

where $[u]^{(0)}=u^{(0)}$ and $0<c_{\text {exp. }}<1$ is the smoothing factor. This algorithm acts as a low-pass filter with cut-off frequency $f_{c}$ (at which the amplitude is reduced by half) approximated by $c_{e x p} \simeq 2 \pi f_{c} \Delta t / \sqrt{3}$ (first-order filter), where $\Delta t$ is the simulation time-step. In our fluid mechanical context, $f_{c}$ should be identified with a "characteristic frequency of the flow". The main advantage of this algorithm is its simplicity, both conceptually and in its implementation.
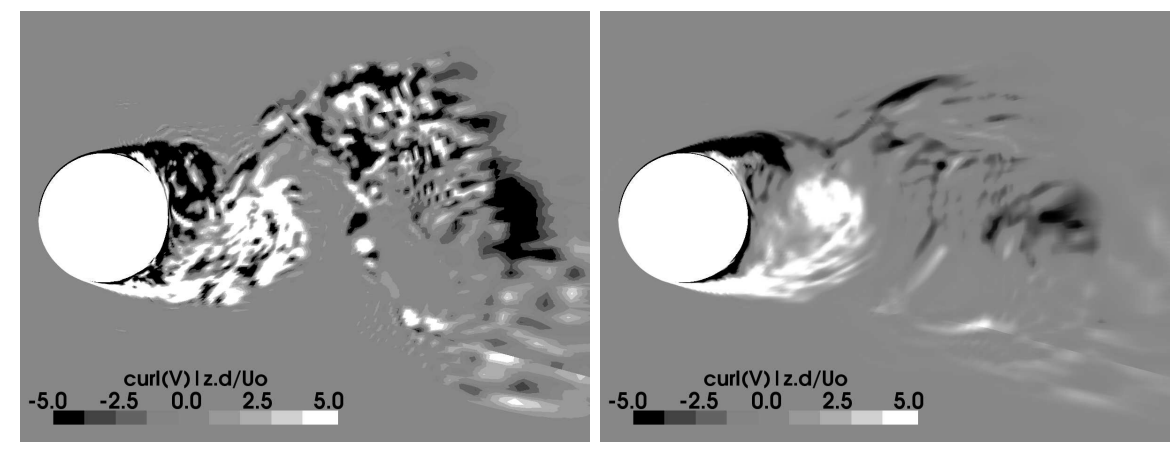

Fig. 1. Cylinder, $R e_{D}=47000$. Left: instantaneous vorticity (non-dimensionnal). Right: vorticity of the exponentially-smoothed flow (non-dimensionnal).

The flow past a circular cylinder at $R e_{D}=47000$ based on diameter $D$ has been examined to test our modeling. In this regime, the flow develops laminar boundary-layers, moving separations, shear-layer transitions and vortex shedding. It is therefore a challenging test-case [7]. The LES was performed by using the Turb'Flow solver [8]. The numerical grid extends over $10 D$ in the radial direction and $3 D$ in the spanwise direction (with a periodic boundary condition). This grid encompasses $3 \times 10^{6}$ mesh-points with a resolution 
$\Delta r^{+} \lesssim 1,(D / 2) \Delta \theta^{+} \lesssim 20$ and $\Delta z^{+} \lesssim 25$ (in wall units) in the turbulent separated region, following standard recommendations for LES [9]. In the smoothing algorithm, $f_{c}$ is fixed at twice the value of the expected vortexshedding frequency. This prescription a priori ensures that large eddies that detach from the cylinder are captured in the mean-flow reconstruction. Indeed, as shown in Fig.1, while the instantaneous flow presents numerous turbulent scales, the smoothed flow mostly captures the vortex shedding.

\begin{tabular}{|c|c|l|}
\hline & simulation & data in literature \\
\hline $\begin{array}{c}\text { St : Strouhal number } \\
\text { of the vortex shedding }\end{array}$ & 0.19 & {$[0.18,0.20]\left(\operatorname{Re}_{D}=4.810^{4}\right)[10]$} \\
$\left.[0.185,0.195]\left(10^{4} \leq R e_{D} \leq 10^{5}\right)[1]\right]$ \\
\hline$\theta_{s}:$ mean separation angle & $88^{\circ}$ & $\simeq 83^{\circ}\left(4.010^{4} \leq R e_{D} \leq 4.510^{4}\right)[11]$ \\
\hline$C_{D}:$ mean drag coefficient & 1.34 & {$[1.0,1.35]\left(R e_{D}=4.810^{4}\right)[10]$} \\
& & $1.35\left(R e_{D}=4.310^{4}\right)[12]$ \\
& & {$[1.0,1.3]\left(R e_{D}=4.810^{4}\right)[13]$} \\
& & {$[1.1,1.3]\left(10^{4} \leq R e_{D} \leq 10^{5}\right)[11]$} \\
\hline$C_{D}^{\prime}:$ rms drag coefficient & 0.09 & {$[0.08,0.1]\left(R e_{D}=4.810^{4}\right)[14]$} \\
& & $0.16\left(R e_{D}=4.310^{4}\right)[12]$ \\
& & {$[0.05,0.1]\left(10^{4} \leq R e_{D} \leq 10^{5}\right)[11]$} \\
\hline$C_{L}^{\prime}:$ rms lift coefficient & 0.77 & {$[0.4,0.8]\left(\operatorname{Re}_{D}=4.810^{4}\right)[14]$} \\
& & {$[0.45,0.55]\left(R e_{D}=4.310^{4}\right)[12]$} \\
& & {$[0.6,0.82]\left(10^{4} \leq R e_{D} \leq 10^{5}\right)[1]$} \\
\hline
\end{tabular}

Table 1. Comparison of flow characteristics with experimental data.

The key flow characteristics are summarized in Table 1. The agreement is good with experimental data, concerning the vortex-shedding frequency and the mean or fluctuating forces. The mean separation angle appears overestimated, but the numerical resolution in the region of the boundary-layer separation is about $2^{\circ}$; the discrepancy is thus only two grid-points. In Fig.2, the angular profile of the mean-pressure coefficient is consistent with experimental data. The angular profile of the root-mean-squared fluctuations of the pressure coefficient is displayed in Fig.3. The overall behavior is well captured, with a maximum around the mean separation angle, but over-estimated. However, the data reported in the figure indicate a dependence on the Reynolds number which may explain, to some degree, the observed discrepancy.

In conclusion, our numerical results demonstrate the good predictive capacity of the method. From a computational viewpoint, this method deserves interests since it is "low-cost" and entirely local in space. It is therefore well adapted for parallelization. A refinement of the smoothing algorithm, in terms of a Kalman filter that adapts its smoothing frequency to the recent history of the signal, is currently investigated.

Acknowledgements: the simulation has been performed by using the local computing facilities, PSMN at ENS-Lyon. 


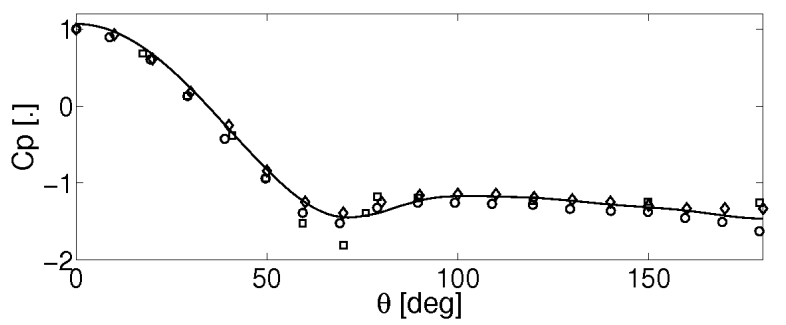

Fig. 2. Mean pressure coefficient around the cylinder. - : simulation at $R e_{D}=$ $4.710^{4}$; ○: exp. data at $R e=4.010^{4}[12] ; \diamond$ exp. data at $R e=4.610^{4}[15] ; \square$ : exp. data at $R e=10^{5}[13]$.

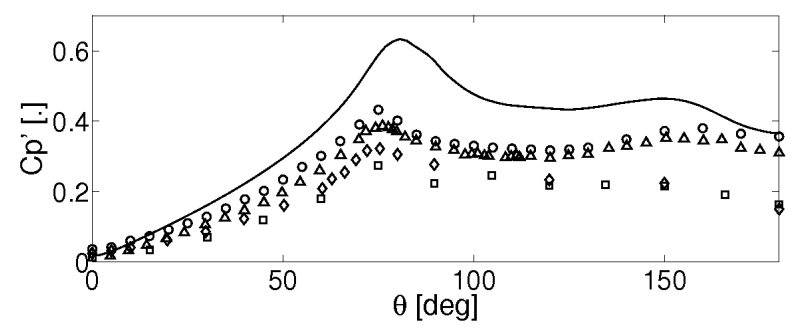

Fig. 3. RMS fluctuations of the pressure coefficient around the cylinder. - : simulation at $R e_{D}=4.710^{4}$; ○: exp. data at $R e=6.110^{4}$ [17]; $\triangle$ : exp. data at $R e=6.110^{4}[19] ; \square$ : exp. data at $R e=10^{5}[18] ; \diamond:$ exp. data at $R e=10^{5}[16]$.

\section{References}

1. E. Lévêque, F. Toschi, L. Shao \& J.-P. Bertoglio, J.Fluid Mech. 570, 491 (2007).

2. L. Shao, S. Sarkar \& C. Pantano, Phys. Fluids 11(5), 1229-1248 (1999).

3. S. Pope, Turbulent Flows, Cambridge University Press, Cambridge, UK (2000).

4. F. Toschi, H. Kobayashi, U. Piomelli \& G. Iaccarino, Proceedings of the Summer Program 2006, Center for Turbulent Research, Stanford, USA (2006).

5. M. Germano et al., Phys. Fluids A, 3, 1760 (1991).

6. J. Smagorinsky, Mon. Weather Rev. 91, 99 (1967).

7. M. Breuer, Heat and Fluid Flow 21, 648 (2000).

8. J. Boudet et al., Journal of Thermal Science, 16(4), 328-336 (2007).

9. P. Sagaut, Large Eddy Simulation for Incompressible Flows, Springer (2001).

10. B. Cantwell and D. Coles. J.Fluid Mech. 136, 321-374 (1983).

11. M. M. Zdravkovich, Oxford University Press (2002).

12. S. Szepessy and P. W. Bearman. J.Fluid Mech. 234, 191-217 (1992).

13. E. Achenbach. J. Fluid Mech. 34, 625-639 (1968).

14. J. H. Gerrard. J.Fluid Mech. 11, 244-256 (1961).

15. E. A. Anderson and A. A. Szewczyk. Exp. in Fluids 23, 161-174 (1997).

16. S. Yokuda and B. R. Ramaprian. Phys. Fluids A 2 (5), 784-791 (1990).

17. H. Nishimura and Y. Taniike. J. Wind Eng. Ind. Aerodyn. 89, 713-723 (2001).

18. J. P. Batham. J. Fluid Mech. 57, 209 (1973).

19. C. Norberg. J. Fluids Struct.. 17, 57-96 (2003). 